\title{
Insulin-binding antibodies in relation to insulin therapy
}

\author{
J. N. HARRIS-JONES, H. MILlER, AND G. OWEN \\ From Llanelly Hospital, Carms., Regional Medical Physics Department, \\ Sheffield, and the Royal Infirmary, Doncaster
}

SYNOPSIS Methods for detecting insulin binding or transporting antibodies are described, involving the use of radioactively labelled insulin (insulin ${ }^{131} \mathrm{I}$ ). These antibodies are detected in a high pro- $\stackrel{i}{\mathrm{~N}}$ portion of patients receiving insulin, particularly those requiring large doses. The protein fractions $\vec{N}$ concerned appear to be the beta and gamma globulins. The significance of the phenomenon is discussed.

The occurrence of insulin binding by the plasma proteins of subjects receiving insulin now seems to have become generally accepted. Methods to demonstrate such binding have included the biological protection methods described by Lowell (1944) and, more recently, methods employing radioactive insulin, such as those reported by Berson, Yalow, Bauman, Rothschild, and Newerly (1956), Burrows, Peters, and Lowell (1957), Kalant, Gomberg, and Schucher (1958), Skom and Talmage (1958), and Miller and Owen (1960a) have been used. The present report is an account of results achieved by the last method, and is based on the examination of a number of sera of patients who have received insulin, mostly for the treatment of diabetes mellitus.

\section{MATERIAL}

Most of the sera examined were from diabetic patients attending the Diabetic Clinic of the Royal Hospital, Sheffield, and the Doncaster Royal Infirmary but in addition a few sera were obtained from schizophrenic patients receiving insulin shock therapy. Blood was collected from these patients in the fasting state before the morning dose of insulin in order to obtain serum with as low an insulin content as possible. Where the patient was in hospital insulin was withheld as long as was considered safe before collecting blood samples. Serum was separated and stored in the frozen state. Patients were chosen to cover a wide range of insulin requirement, but those requiring a daily dose of 100 units or more were considered of particular interest.

METHODS

Insulin was labelled with the isotope iodine-131 by the Received for publication 13 June 1962. methods of Pearson (1959) or McFarlane (1958). The $\stackrel{\widehat{S}}{S}$ methods used for the detection of insulin binding in this investigation have already been published (Miller and $\mathscr{Q}$ Owen, 1960a); in principle they have followed the techniques described by Berson et al. (1956) with the $\square$ addition of immuno-electrophoretic methods described by Miller and Owen $(1960 \mathrm{~b}, 1961)$. Briefly they are as follows:-

ZONE ELECTROPHORESIS Electrophoretic strips of the test sera previously incubated with insulin ${ }^{131} \mathrm{I}$ were $\overrightarrow{\vec{A}}$ prepared on an acetylated cellulose medium. This $\frac{0}{3}$ medium allows free mobility of the insulin which is not $\vec{\partial}$ absorbed at the point of application. Measurements of activity along the strip were made with a Geiger counter behind a narrow slot. Autoradiographs were then pre-용 pared and measurements made of the distribution of $\sigma$ photographic density along the autoradiograph using a densitometer with a $2 \mathrm{~mm}$. illuminated spot. A calibration ${ }^{\circ}$ curve was drawn to allow the density distribution to be 3 interpreted in terms of activity in the original zone electrophoresis strip (Miller and Owen, 1960a). Experience $J$ showed that it was desirable to use excess radioactive $D$ insulin to saturate and maintain saturation of the insulin-binding proteins. The excess unbound radio- $N$ insulin moves freely with a characteristic mobility which is approximately that of the alpha-1 globulin. The bound $N$ insulin appears in the beta-gamma globulin region of the $N$ electrophoresis strip.

Figure 1 illustrates the manner in which radioactive 2 insulin is first bound by the antibody beta-gamma globulins, and the unbound insulin available after the $\bar{\Phi}$ saturation of these globulins moves approximately to the ${ }^{+}$ alpha-1 globulin position. In this diagram the distribution of radioactivity on four electrophoretic strips is plotted. These strips have been run with a serum from an insulin- $\stackrel{\mathbb{D}}{\Omega}$ treated diabetic patient incubated with different amounts $\frac{\mathbb{D}}{8}$ of the radioactively labelled insulin. In each case the sample was applied at the point corresponding to $0 \mathrm{~cm}$. 


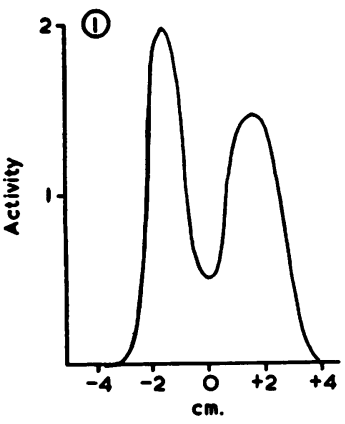

$0.2 \mathrm{ml}$. serum + $0.34 \mu \mathrm{g}$. $\left.13\right|_{1}$ insulin

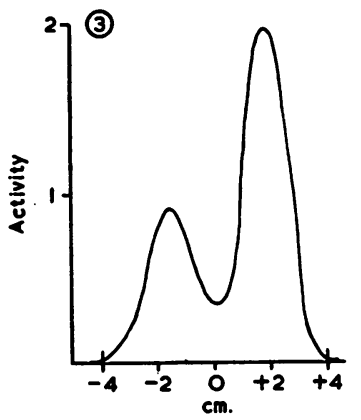

$0.2 \mathrm{ml}$ serum $+1.36 \mu \mathrm{g}$ $|3| \mathbf{f}$ insulin
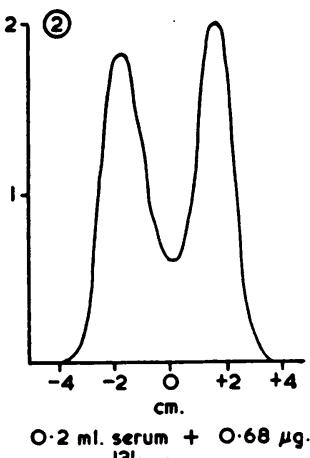

$\left.13\right|_{1}$ insulin

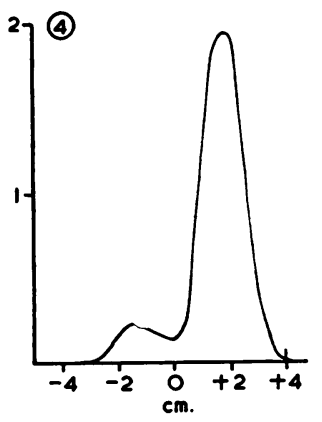

$0.2 \mathrm{ml}$ serum $+5.45 \mu \mathrm{g}$.

$\left.13\right|_{1}$ insulin
FIG. 1. Saturation of binding capacity. Activity distributions along zone electrophoresis strips for sample of serum incubated with varying amounts of radioactive insulin. The patient was receiving 140 units of insulin per day.

on the horizontal scale and the albumin and alpha-1 globulins run in the positive direction, that is, the peak on the curves at approximately $+1.5 \mathrm{~cm}$. represents free insulin. The bound insulin is represented by the peak at approximately $-1.5 \mathrm{~cm}$. The binding capacity of the serum is limited and with increasing amounts of added insulin ${ }^{131}$ I a smaller proportion of the radioactivity appears in the bound fraction.
An estimate of the binding capacity can be made from the distribution of radioactivity along the electrophoresis strips as described by Miller and Owen (1960a). This estimate is based on a measurement of the ratio of the insulin bound by the beta-gamma globulins to the total insulin added to the serum sample.

IMMUNOELECTROPHORESIS The resolution obtained with zone electrophoresis was often somewhat inadequate and information on the precise sites of binding was thus somewhat unsatisfactory. To provide clearer information as to which of the serum proteins were responsible for binding insulin immunoelectrophoresis was adopted. Insulin ${ }^{131}$ I was again used so that autoradiographs of the immunoelectrophoretic strips would clearly demonstrate sites of radioactivity which were then related to the original immunoelectrophoretogram for identification of the particular plasma proteins responsible for the binding. Details of this technique are given by Miller and Owen (1960b; 1961).

A typical result of the immunoelectrophoresis technique is illustrated in Figure 2. The diagram shows the results of the immunoelectrophoresis on an agar plate. The left-hand section shows the stained precipitation lines. The original serum sample from the treated diabetic containing the labelled insulin is placed in the two small holes in the agar. Electrophoresis separation is performed with the positive potential at the right-hand end of the plate. For the development of the precipitin reaction thin strips of filter paper have been placed along the top and bottom of the agar plate and a third strip between the two holes. The first two strips were soaked in Pasteur horse anti-human serum and the third strip soaked in anti S7 gamma globulin. The right-hand section of the diagram shows the autoradiograph of the precipitation pattern.

\section{RESULTS}

Immunoelectrophoretic techniques have demonstrated binding in both beta and gamma globulins. More recently improved resolution in the zone electrophoresis on cellulose acetate, combined with the use of a higher specific activity of the tagged insulin, has enabled the binding by beta and gamma

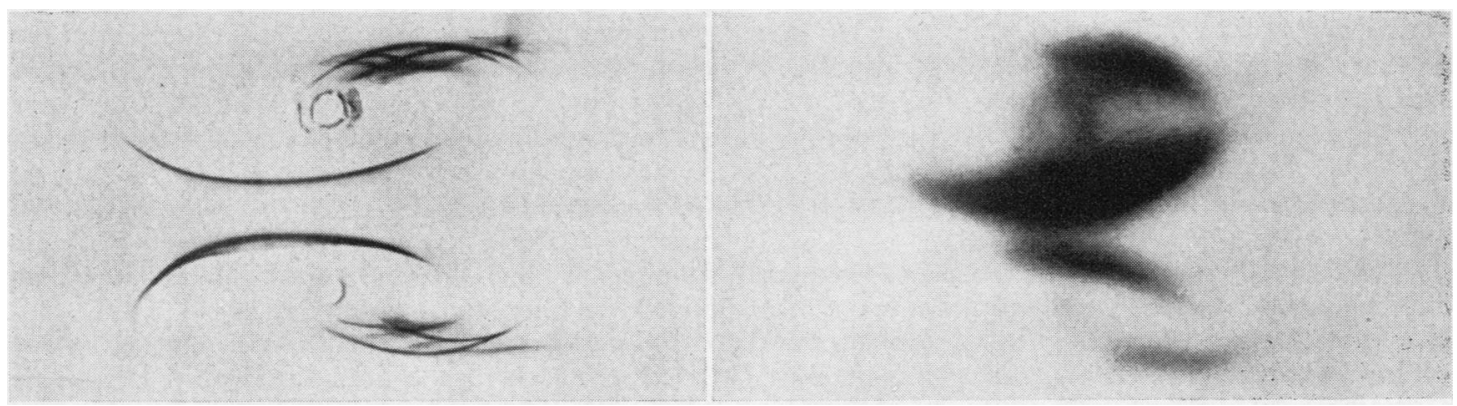

FIG. 2. Autoradiography of immunoelectrophoresis patterns. The distribution of radioactivity demonstrates the binding of the labelled insulin in both beta and gamma globulins. 


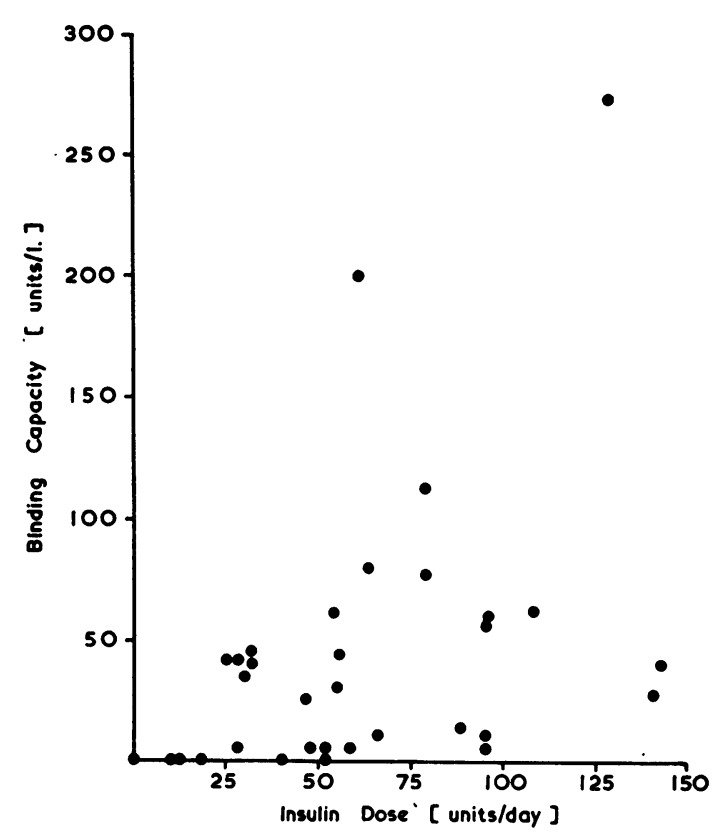

FIG. 3. Relation between binding capacity per litre of serum and the maximum daily insulin requirement.

globulins to be demonstrated by this technique also. Similar results have been obtained in experimentally produced animal antisera and in this case three sites of binding in the beta-gamma globulins were demonstrated using zone electrophoresis on cellulose acetate.

Repeated examinations were made on the sera of some 50 patients receiving insulin in varying doses, and evidence of significant binding sought by the techniques described above. Insulin binding was detected in the majority of these sera, and an attempt was made to relate the amount of insulin bound by antibody with the daily insulin requirement of the patient.

The results of the survey of binding capacity in the sera tested are shown in Figure 3. The relation between the binding capacity in units of insulin per litre of serum and the maximum daily insulin requirement of the patient is illustrated.

\section{DISCUSSION}

The binding of insulin by the beta and gamma globulins appears to be a true antibody function of these proteins as the phenomenon is related to insulin administration and not to diabetes mellitus. In common with others we have found significant binding in the serum of insulin-treated schizophrenics. The sera we have studied are from diabetic patients whose insulin requirements have ranged up to about 150 units per day, and the results parallel those originally reported by Berson et al. (1956), in $\overrightarrow{\vec{s}}$ that insulin binding may be detected in the serum of $\frac{9}{0}$ the majority of patients receiving insulin, and that it is not confined to insulin-resistant patients as $\frac{\bar{\sigma}}{\bar{c}}$ suggested by Burrows et al. (1957). Results obtained $\vec{\widetilde{ }}$ in this investigation indicate that whereas insulin $\varrho$ binding can be shown neither to be the cause of high 0 insulin requirement, nor to be invariably associated $\vec{\circ}$ with it, nevertheless high insulin binding is to be found with increasing frequency in those patients $\vec{\sigma}$ who have the largest insulin requirements. This being so, it might be postulated that the relatively? high dose of insulin needed by many diabetic $\vec{\sigma}$ patients may in part be necessary because before? any insulin is available to satisfy physiological needs $\vec{N}$ a large amount must be first supplied to saturate $\circ$ the binding antibodies. It was hoped to explore the possibility of explaining the increase in insulin $\vec{z}$ requirements which frequently accompanies pyogenic infections in diabetic subjects by the demon- $\frac{\hat{J}}{\sigma}$ stration of a non-specific rise in the globulin fractions $\vec{\varphi}$ and therefore of an increase in insulin binding. Suitable material for such a study was not available, but significant binding was demonstrated in the serum of a young female diabetic with pneumonia. This patient's binding capacity fell from 61 units per litre to 24 units per litre following recovery from her: infection.

The precise site of insulin binding has been the $\overrightarrow{\hat{O}}$ subject of some discussion amongst investigators in 3 this field. In most reports of investigations with labelled insulin in vitro it has been stated that insulin is bound by the gamma globulin fraction (Weiger and Colwell, 1956; Burrows et al., 1957; Kalant et al., 1958). Berson and Yalow (1957) 3 thought that the inter beta-gamma zone was probably the site of insulin binding. Serum removed after the intravenous injection of labelled insulin appears to ${ }_{0}$ confirm that binding occurs on the gamma globulin. Ultracentrifuge studies of serum from insulin-treatedo subjects incubated with labelled insulin seem to confirm this observation (Berson et al., 1956). $\sigma$ Miller and Owen (1960b), however, by using N autoradiographs of immunoelectrophoretic patterns N of the serum of insulin-treated subjects incubated $\omega$ with insulin ${ }^{131}$ I have shown distinct bands of activity at both the gamma and beta globulin 0 fractions in which the binding at the latter site would appear occasionally to be the greater.

Other globulins associated with insulin have been $\frac{T}{0}$ described, such as the antibody reported as being $\vec{\otimes}$ responsible for insulin skin reactions in the beta $\frac{\odot}{\mathrm{O}}$ fraction (Loveless and Cann, 1955), and an insulin antagonist found in the presence of ketosis associated 
with the alpha-1 globulin (Field, Tietze, and Stetten, 1957). An insulin antagonist present in normal subjects and insulin-treated diabetics has been demonstrated in the albumin fraction by VallanceOwen, Dennes, and Campbell (1958).

\section{CONCLUSION}

The results of this investigation are in general agreement with those of other workers, particularly where the same techniques have been used. It seems reasonable to conclude that there may be demonstrated in the serum of patients treated with insulin an insulinbinding antibody. Although this antibody appears in response to insulin therapy, in general it is found most frequently in those subjects who require large doses of insulin. Whereas it may be said that a relationship exists between these two factors, results in individual cases show it not to be invariable. It would appear tempting to suppose that the higher insulin dosage required by some patients is necessary to saturate these binding antibodies; methods at present available for estimating the amount of insulin bound have failed to show a direct relationship between insulin-binding capacity and the dose of insulin required. High insulin-binding capacities have been demonstrated in insulin-resistant patients by Berson and Yalow (1959). Despite views to the contrary, it now seems to have been demonstrated with some degree of certainty that insulin binding may be a function of both the beta and gamma globulins, and it may yet be shown that this differential binding may be of significance in terms of insulin requirement or resistance.

We are grateful for samples of sera provided by Dr. C. E. Davies, of the Royal Hospital, Sheffield, and by Dr. P. Milligan and Dr. L. A. Hawkins, of the Doncaster Royal Infirmary. We also wish to acknowledge the technical assistance of Miss N. Brammer of the Regional Medical Physics Department, Sheffield.

\section{REFERENCES}

Berson, S. A., Yalow, R. S., (1957). Ibid., 36, 642.

- _ (1959). Ibid., 38, 2017.

-, Bauman, A., Rothschild, M. A., and Newerly, K. (1956). J. clin. Invest., 35, 170.

Burrows, B. A., Peters, T., and Lowell, F. C. (1957). Ibid., 36, 393.

Field, J. B., Tietze, F., and Stetten, D. Jr. (1957). Ibid., 36, 1588.

Kalant, N., Gomberg, C., and Schucher, R. (1958). Lancet, 2, 614.

Lowell, F. C. (1944). J. clin. Invest., 23, 233.

Loveless, M. H., and Cann, J. R. (1955). J. Immunol., 74, 329.

McFarlane, A. S. (1958). Nature (Lond.), 182, 53.

Miller, H., and Owen, G. (1960a). Radioaktive Isotope in Klinik und Forschung Suppl. to Strahlentherapie), 4, 266. (1960b). Nature (Lond.), 188, 67.

- (1961). Vox. Sang. (Basel), 6, 190.

Pearson, J. D. (1959). Lancet, $1,967$.

Skom, J. H., and Talmage, D. W. (1958). J. clin. Invest., 37, 783.

Vallance-Owen, J., Dennes, E., and Campbell, P. N. (1958). Lancet, $2,336$.

Weiger, R. W., and Colwell, A. R. (1956). Clin. Res. Proc., 4, 123. 